\title{
Decision consultations on preoperative radiotherapy for rectal cancer: large variation in benefits and harms that are addressed
}

\author{
M Kunneman*,1, C A M Marijnen ${ }^{2}$, T Rozema ${ }^{3}, \mathrm{H} \mathrm{M} \mathrm{Ceha}^{4}$, D A R H Grootenboers ${ }^{5}$, K J Neelis ${ }^{2}$,
} A M Stiggelbout ${ }^{1}$ and A H Pieterse ${ }^{1}$

${ }^{1}$ Leiden University Medical Center, Department of Medical Decision Making, PO Box 9600, 2300 RC Leiden, The Netherlands; ${ }^{2}$ Leiden University Medical Center, Department of Clinical Oncology, PO Box 9600, 2300 RC Leiden, The Netherlands; ${ }^{3}$ Verbeeten Institute, PO Box 90120, 5000 LA Tilburg, The Netherlands; ${ }^{4}$ Radiotherapy Center West, Medical Center Haaglanden, PO Box 432, 2501 CK The Hague, The Netherlands and ${ }^{5}$ Reinier de Graaf Group, Department of Radiotherapy, PO Box 5011,2600 GA Delft, The Netherlands

Background: For shared decision making to be successful, patients should receive sufficient information on possible benefits and harms of treatment options. The aim of this study was to evaluate what information radiation oncologists provide during the decision consultation about preoperative radiotherapy with rectal cancer patients.

Methods: Decision consultations of 17 radiation oncologists with 81 consecutive primary rectal cancer patients, eligible for shortcourse radiotherapy followed by a low-anterior resection, were audio taped. Tapes were transcribed and analysed using the ACEPP (Assessing Communication about Evidence and Patient Preferences) coding scheme.

Results: A median of seven benefits/harms were addressed per consultation (range, 2-13). This number ranged within and between oncologists and was not clearly associated with the patient's characteristics. A total of 30 different treatment outcomes were addressed. The effect of radiotherapy on local control was addressed in all consultations, the effect on survival in $16 \%$. The most important adverse effects are bowel and sexual dysfunction. These were addressed in $82 \%$ and $85 \%$ of consultations, respectively; the latter significantly less often in female than in male patients. Four out of five patients did not initiate discussion on any benefits/harms.

Conclusions: Our results showed considerable inconsistency between and within oncologists in information provision, which could not be explained by patient characteristics. This variation indicates a lack of clarity on which benefits/harms of radiotherapy should be discussed with newly-diagnosed patients. This suboptimal patient information hampers the process of shared decision making, in which the decision is based on each individual patients' weighing of benefits and harms.

In 2012, about 380000 new cases of rectal cancer were diagnosed worldwide, and this number is increasing annually (Cancer Research UK, 2014). Primary treatment consists of total mesorectal excision. The effect of short-course $(5 \times 5 \mathrm{~Gy})$ preoperative radiotherapy (PRT) on local control in patients with localised disease has been clearly demonstrated (van Gijn et al, 2011). However, difficulties arise in selecting those patients who benefit most from
PRT, with a high number needed to treat to prevent one local recurrence (Stephens et al, 2010; van Gijn et al, 2011). Furthermore, PRT is associated with adverse effects, the most important of which are bowel problems and sexual dysfunction (Birgisson et al, 2007; Stephens et al, 2010).

Large differences exist between individual rectal cancer-patients' treatment preferences and their valuation of possible benefits and

*Correspondence: M Kunneman; E-mail: Kunneman@lumc.nl

Received 28 May 2014; revised 23 September 2014; accepted 25 September 2014; published online 21 October 2014 
harms of treatment (Pieterse et al, 2007). These preferences cannot be predicted based on socio-demographic factors or disease characteristics (Pieterse et al, 2011). This situation, in which individual patients weigh possible benefits and harms of treatment differently, is highly suitable for shared decision making (MullerEngelmann et al, 2011; Wong and Szumacher, 2012).

In general, rectal cancer-treatment guidelines make little or no recommendations on which benefits and harms to communicate to patients. The Dutch guidelines on colorectal cancer state that clinicians should 'discuss the possible benefits and harms of radiotherapy with the patient', without specifying which benefits and harms (Comprehensive Cancer Centre the Netherlands, 2014). Informing patients about possible treatment options and associated outcomes is a minimal and necessary condition for eliciting and considering patient preferences and for involving them in treatment decision making. Moreover, it helps to meet cancer patients' information needs (Douma et al, 2012). Patients who are well-informed and have a clear understanding of their preferences regarding treatment outcomes, experience less anxiety (Glavassevich et al, 1989). In addition, appropriate and timely information can help provide better management of cancertreatment side effects, and thereby reduce physical side effects (Dunn et al, 2004; Guleser et al, 2012). The majority of treated cancer patients, however, indicate that they are not or not sufficiently informed about possible harms of the treatment they underwent (Nicolaije et al, 2012).

This study aimed to evaluate the information provision during the first consultation between radiation oncologists and rectal cancer patients, in which the decision about PRT is usually made. Research questions to be answered were: (1) Which benefits and harms of PRT are addressed in the consultation? (2) Are benefits and harms addressed on the initiative of the radiation oncologist, the patient or a companion? and (3) If variation in the benefits and harms addressed or in the initiation of these is seen, is this variation associated with patient characteristics?

\section{MATERIALS AND METHODS}

Study population. This study was conducted in 4 of the 18 radiotherapy centers in the Netherlands. All primary rectal cancer patients eligible for short-course $(5 \times 5 \mathrm{~Gy})$ PRT followed by a low-anterior resection, with a good comprehension of the Dutch language, were eligible for inclusion.

All radiation oncologists treating rectal cancer patients were asked to participate.

Procedure. Inclusion of patients started in one radiation center and was gradually extended to the other centers. Decision consultations of participating radiation oncologists with all consecutive eligible primary rectal cancer patients scheduled to undergo a low-anterior resection were audio taped. The Medical Ethics Committee of Leiden University Medical Center approved the study. Eligible patients signed an informed-consent form and completed a self-report questionnaire to assess socio-demographic details before the consultation started. Radiation oncologists were asked to fill in a questionnaire assessing their socio-demographic and work-related details at the start of the study.

Measures. Audio tapes of consultations were transcribed verbatim and analysed using the ACEPP (Assessing Communication about Evidence and Patient Preferences) coding scheme (Shepherd et al, 2011). By using this scheme, all health related benefits and harms of PRT that were discussed in the consultation were identified. Benefits and harms related to inconvenience or costs, such as travel time or expenses, were not included. Two raters independently coded the same 10 (12\% of total number) audio tapes. Inter-rater reliability was high (Cohen's $K=0.83$; Landis and Koch, 1977).
The remaining tapes were coded individually (intra-rater agreement based on eight (10\%) tapes per rater coded twice with a time difference of 19 months, Cohen's $K=0.78-0.85$ ).

Statistical analyses. Descriptive statistics were used to report patients' and radiation oncologists' characteristics, and information provision on benefits and harms of PRT. The number of benefits and harms addressed per consultation were not normally distributed, so medians are presented and compared with MannWhitney U-tests. Spearman correlations were used to measure linear dependence between number of benefits/harms addressed and consultation time. A logistic regression analysis was conducted to predict the discussion of benefits/harms, using age as a predictor. Using $\chi^{2}$ tests, initiative of patients and clinicians to discuss benefits and harms was compared. Significance testing was done two-sided at $\alpha=0.05$.

\section{RESULTS}

Participants. In total, 112 eligible patients, diagnosed between November 2010 and March 2013, were asked to participate. Of them, 84 agreed (response rate $75 \%$ ). Three patients were excluded from the analyses because their consultation had not (completely) been recorded.

All 17 radiation oncologists treating patients with rectal cancer agreed to participate and audio taped a median of five consultations with new rectal cancer patients (range, 1-11).

In Table 1 patient and clinician characteristics are listed.

Benefits and harms addressed per consultation. Figure 1 shows the variation in the number of benefits and harms addressed per consultation both between and within radiation oncologists. Overall, a median of seven benefits and harms were addressed per consultation (range, 2-13). There was no significant association between the number of benefits and harms addressed and patient gender, age, or educational level.

Overall, consultations lasted significantly longer when more benefits and harms were addressed (median $=33 \mathrm{~min}$ for $\leqslant 6$ benefits/harms vs $40 \mathrm{~min}$ for $\geqslant 7$ benefits/harms, rho $=0.23$, $P<0.05)$.

In the 81 audio taped consultations, a total of 30 different benefits and harms of PRT in rectal cancer were addressed (see Figure 2). The beneficial effect of PRT on local control of the

\section{Table 1. Participant characteristics}

\begin{tabular}{|c|c|}
\hline & $N$ \\
\hline \multicolumn{2}{|l|}{ Patients $(\mathbf{N}=\mathbf{8 1})$} \\
\hline Mean age, years \pm s.d. (range) & $65 \pm 10.4(40-87)$ \\
\hline Male & $57(70 \%)$ \\
\hline Partner (yes) ${ }^{a}$ & $51(76 \%)$ \\
\hline \multicolumn{2}{|l|}{ Educational level ${ }^{a, b}$} \\
\hline Low & $20(30 \%)$ \\
\hline Intermediate & $30(45 \%)$ \\
\hline High & $16(24 \%)$ \\
\hline Companion in consultation & $73(90 \%)$ \\
\hline \multicolumn{2}{|l|}{ Clinicians $(N=17)$} \\
\hline Mean age, years \pm s.d. (range) & $39 \pm 6.3(27-50)$ \\
\hline Male & $5(29 \%)^{c}$ \\
\hline Median time since specialisation, years (range) & $4(0-20)$ \\
\hline Median number of rectal cancer patients per month (range) & $3(1-8)$ \\
\hline \multicolumn{2}{|c|}{ 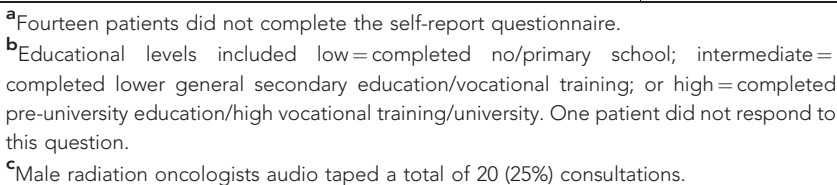 } \\
\hline
\end{tabular}




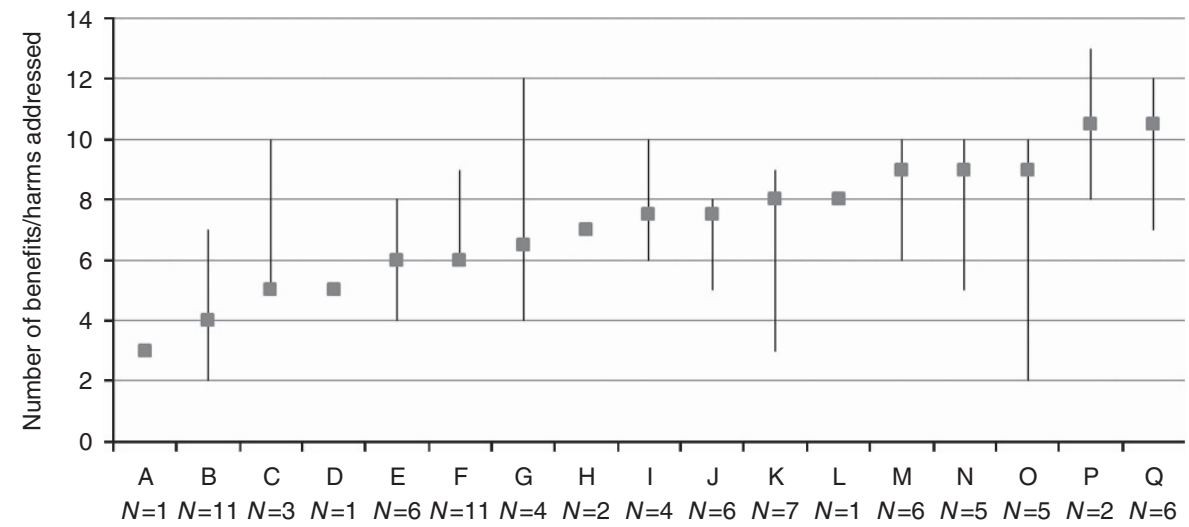

Radiation oncologist

Figure 1. Number (median and range) of benefits and harms addressed in consultations per radiation oncologist, sorted by median. Abbreviation: $\mathrm{N}=$ number of consultations taped per radiation oncologist.

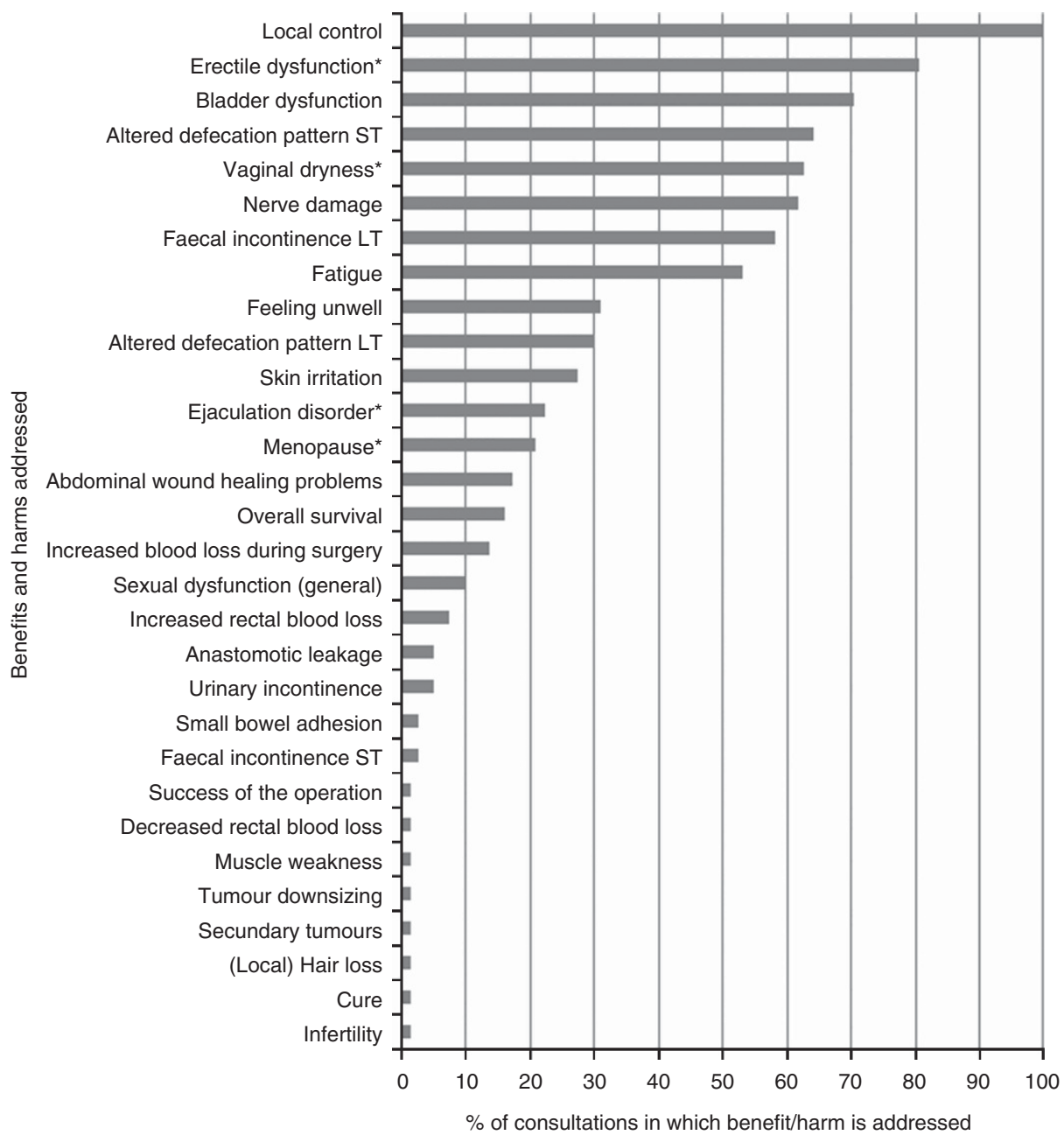

Figure 2. Benefits and harms of PRT addressed in decision consultations. Abbreviations: ST= short term; LT =long term. *As a percentage of consultations with patients from relevant patient group (male/female patients).

cancer was addressed in all consultations. The effect of PRT on overall survival was addressed in 13 (16\%) consultations, conducted by five (29\%) different radiation oncologists.

The most important adverse effects of PRT described in the literature are bowel problems and sexual dysfunction. Bowel problems, such as altered defecation pattern, faecal incontinence or rectal blood loss, were addressed in $66(82 \%)$ consultations, conducted by 15 (88\%) different radiation oncologists. In 53 (65\%) consultations short-term bowel problems during treatment were discussed, and in 57 (70\%) consultations long-term bowel problems were discussed, with a high within-patient overlap. There was no significant association between discussing bowel problems and patient gender, age, or educational level.

Long-term sexual dysfunction, such as erectile or ejaculation disorders (male patients), vaginal dryness (female patients), or sexual problems in general (without further specification) were 
addressed in $69(85 \%)$ consultations, conducted by $16(94 \%)$ different radiation oncologists, and significantly less often in female than in male patients $(N=16,67 \%$ vs $N=53,93 \%$ respectively; $\left.\chi^{2}=7.56, \quad P<0.01\right)$. Although not statistically significant, the older the patients were, the less often sexual dysfunction was discussed during the consultation $(P=0.07)$. There was no association between discussing sexual dysfunction and patient's educational level or marital status.

Initiative to address benefits and harms. Across consultations, radiation oncologists initiated $89 \%$ of the discussions about benefits and harms. The other discussions were initiated by the patients ( $9 \%$ of benefits/harms) or the patients' companions ( $2 \%$ of benefits/harms), for example by asking a question or addressing a new topic. In total, there were 16 patients (20\%) who showed initiative during their consultation to discuss at most two harms (e.g., skin irritation, feeling unwell, bladder dysfunction, long-term faecal incontinence, fatigue, nerve damage, secondary tumours, muscle weakness, and abdominal wound healing problems). Topics that companions additionally raised were anastomotic leakage, overall survival, and sexual dysfunction.

In consultations in which the patient initiated the discussion of a harm, a median of two more benefits/harms were addressed compared to consultations with more passive patients (median $=8$ vs $6, P<0.05)$. There was no significant association between whether or not a patient took the initiative to raise a topic and patient's gender, age or education level, or being accompanied during the consultation.

\section{DISCUSSION}

This study aimed to examine what information is provided about possible benefits and harms of PRT in the first consultation between newly-diagnosed rectal cancer patients and their radiation oncologist, in which a decision about PRT is usually made.

Our study showed considerable variation, both in the number and in the type of benefits and harms that were discussed. This variation was present between as well as within radiation oncologists and could not consistently be explained by patients' characteristics. The variation found implies that some patients receive limited information, while other patients are informed extensively. It is a necessary condition for informed consent and shared decision making alike, that all patients receive sufficient information on possible benefits and harms of treatment options (Stiggelbout et al, 2012). At the same time, the more information is given, the less patients usually remember (McGuire, 1996). Depending on the total amount of information given, it is expected that about $40 \%-80 \%$ of this information is forgotten immediately after the consultation (Kessels, 2003), though this percentage should decrease when clinicians tailor their information to patients' frame of reference (Tuckett et al, 1985; Colagiuri et al, 2012). Another potential drawback of extending information given to patients, is that placebo research in other settings have shown that patients tend to report experiencing side effects they have been warned about (Colagiuri et al, 2012). These caveats do not imply that information should not be provided, but rather highlight the importance of consensus about which benefits and harms should be presented to newly-diagnosed rectal cancer patients.

The beneficial effect of PRT on local control was addressed in all consultations. In contrast, the effect on overall survival was discussed in a small portion of consultations only. This finding might not come as a surprise given that PRT does not improve overall survival in this patient group (van Gijn et al, 2011). However, if the absence of a survival benefit is not made explicit in the consultation, many patients might wrongly assume that increased local control will result in increased overall survival.
Discussing both topics prevents patients from interpreting information wrongly, and will help them develop realistic expectations on the possible benefit of treatment.

PRT is associated with several adverse effects, such as bowel and sexual dysfunction (Birgisson et al, 2007). Both topics were addressed in the majority of consultations, but over one in four patients did not receive information on the effect of PRT on longterm bowel dysfunction, whilst half of irradiated-rectal cancer patients will experience some form of faecal incontinence. Sexual dysfunction was addressed in a large majority, however more often with male than with female patients. Because of the high prevalence of in particular long-term bowel and sexual dysfunction in patients treated with PRT, and the lack of gain in overall survival, our findings show that there is still much room for improvement in information provision. Furthermore, if these topics are not discussed during the consultation, the trade-off between possible benefits and harms as the basis for the treatment recommendation might not be clear to the patient.

It is noteworthy that radiation oncologists occasionally addressed benefits or harms which have not been described in the literature (e.g., increased or decreased rectal blood loss) or which do not hold for short-course $(5 \times 5 \mathrm{~Gy})$ PRT (e.g., tumour downsizing). This highlights the need for a core list of topics to be addressed or not during the consultation with newly-diagnosed rectal cancer patients. In a follow-up study, we intend to seek consensus between rectal cancer patients and radiation oncologists on which benefits and harms of PRT should be addressed with all newly-diagnosed rectal cancer patients during the decision consultation. This follow-up study will result in a core list of topics that need to be addressed. As the national treatment guidelines are a reference for clinicians, the core list will be included in the revised national guidelines on colorectal cancer. Further implementation strategies, such as the use of communication checklists or leaflets in addition to the oral communication, need to be considered in the future.

Radiation oncologists initiated the discussion of most of the benefits and harms addressed. About four out of five patients did not initiate discussion on any benefits/harms. It has been shown that cancer patients are often unsure about what they should ask their clinician, (Dimoska et al, 2008; Brown et al, 2011), but the lack of patients' initiative could also imply that they perceive their radiation oncologists to be comprehensive. When patients actually take the initiative to discuss outcomes of treatment, significantly more benefits and harms were discussed in the consultation. This implies that outcomes that the patient brings forward add to the outcomes that the clinician already addresses.

A limitation of this study is that we present quantitative data. Conclusions cannot be drawn about the quality of information provision on benefits and harms of PRT, nor about the consistency of quality between and within radiation oncologists. Furthermore, because of relatively small numbers of patients included per radiation oncologist, we were unable to assess associations between the variation in benefits and harms discussed and oncologists' characteristics. Finally, it is noteworthy that we only have data on information provision during patient's consultations with the radiation oncologist, and not during earlier consultations with other clinicians, such as the surgeon or the gastroenterologist. Future research should focus on establishing whether information provision is consistent and sufficient across specialties.

In conclusion, our results showed considerable variation in information provision during the decision consultation on PRT regarding possible benefits and harms of PRT in rectal cancer. This variation indicates a lack of clarity on which benefits and harms of PRT should be discussed with a newly-diagnosed patient. Radiation oncologists should be aware of this between- and within-clinician variation. Standardizing information provision and making sure that all relevant benefits and harms are discussed 
with each individual patient would not only help to meet patients' information needs, it would also promote a process of shared decision making about radiotherapy, in which treatment decisions are a function of individual patients' weighing of benefits and harms.

\section{ACKNOWLEDGEMENTS}

We thank all participating patients and radiation oncologists for their efforts. We thank the Dutch Cancer Society for their support (UL 2009-4431). Study sponsors had no involvement on the study design, in the collection, analysis and interpretation of the data, in the writing of the manuscript, and in the decision to submit the manuscript for publication.

\section{REFERENCES}

Birgisson H, Pahlman L, Gunnarsson U, Glimelius B (2007) Late adverse effects of radiation therapy for rectal cancer-a systematic overview. Acta Oncol 46(4): 504-516.

Brown RF, Butow PN, Juraskova I, Ribi K, Gerber D, Bernhard J, Tattersall MH (2011) Sharing decisions in breast cancer care: development of the Decision Analysis System for Oncology (DAS-O) to identify shared decision making during treatment consultations. Health Expect 14(1): 29-37.

Cancer research UK (2014) http://www.cancerresearchuk.org/cancer-info/ cancerstats/world/.

Colagiuri B, McGuinness K, Boakes RA, Butow PN (2012) Warning about side effects can increase their occurrence: an experimental model using placebo treatment for sleep difficulty. J Psychopharmacol 26(12): 1540-1547.

Comprehensive cancer centre the Netherlands (2014) Guidelines for the management of colorectal cancer and colorectal liver metastases.

Dimoska A, Tattersall MH, Butow PN, Shepherd H, Kinnersley P (2008) Can a "prompt list" empower cancer patients to ask relevant questions? Cancer 113(2): 225-237.

Douma KF, Koning CC, de Haes HC, Zandbelt LC, Stalpers LJ, Smets EM (2012) Do radiation oncologists tailor information to patients needs? And, if so, does it affect patients? Acta Oncol 51(4): 512-520.

Dunn J, Steginga SK, Rose P, Scott J, Allison R (2004) Evaluating patient education materials about radiation therapy. Patient Educ Couns 52(3): 325-332.

Glavassevich M, Thomas S, Galloway SC (1989) Informational needs of patients who undergo excision of an acoustic neuroma. Axone 11(1): 17-19.

Guleser GN, Tasci S, Kaplan B (2012) The experience of symptoms and information needs of cancer patients undergoing radiotherapy. J Cancer Educ 27(1): 46-53.
Kessels RP (2003) Patients' memory for medical information. J R Soc Med 96(5): 219-222.

Landis JR, Koch GG (1977) The measurement of observer agreement for categorical data. Biometrics 33(1): 159-174.

McGuire LC (1996) Remembering what the doctor said: organization and adults' memory for medical information. Exp Aging Res 22(4): 403-428.

Muller-Engelmann M, Keller H, Donner-Banzhoff N, Krones T (2011) Shared decision making in medicine: the influence of situational treatment factors. Patient Educ Couns 82(2): 240-246.

Nicolaije KA, Husson O, Ezendam NP, Vos MC, Kruitwagen RF, Lybeert ML, van de Poll-Franse LV (2012) Endometrial cancer survivors are unsatisfied with received information about diagnosis, treatment and follow-up: a study from the population-based PROFILES registry. Patient Educ Couns 88(3): 427-435.

Pieterse AH, Stalmeier PF, Kroep JR, Stiggelbout AM (2011) Adjuvant cancer treatment: what benefit does the patient consider worthwhile? Ned Tijdschr Geneeskd 155(45): A3905.

Pieterse AH, Stiggelbout AM, Baas-Thijssen MC, van de Velde CJ, Marijnen CA (2007) Benefit from preoperative radiotherapy in rectal cancer treatment: disease-free patients' and oncologists' preferences. Br J Cancer 97(6): 717-724.

Shepherd HL, Barratt A, Trevena LJ, McGeechan K, Carey K, Epstein RM, Butow PN, Del Mar CB, Entwistle V, Tattersall MH (2011) Three questions that patients can ask to improve the quality of information physicians give about treatment options: a cross-over trial. Patient Educ Couns 84(3): 379-385.

Stephens RJ, Thompson LC, Quirke P, Steele R, Grieve R, Couture J, Griffiths GO, Sebag-Montefiore D (2010) Impact of short-course preoperative radiotherapy for rectal cancer on patients' quality of life: data from the Medical Research Council CR07/National Cancer Institute of Canada Clinical Trials Group C016 randomized clinical trial. J Clin Oncol 28(27): 4233-4239.

Stiggelbout AM, van der Weijden T, De Wit MP, Frosch D, Legare F, Montori VM, Trevena L, Elwyn G (2012) Shared decision making: really putting patients at the centre of healthcare. BMJ 344: e256.

Tuckett DA, Boulton M, Olson C (1985) A new approach to the measurement of patients' understanding of what they are told in medical consultations. J Health Soc Behav 26(1): 27-38.

van Gijn W, Marijnen CA, Nagtegaal ID, Kranenbarg EM, Putter H, Wiggers T, Rutten HJ, Pahlman L, Glimelius B, van de Velde CJ (2011) Preoperative radiotherapy combined with total mesorectal excision for resectable rectal cancer: 12-year follow-up of the multicentre, randomised controlled TME trial. Lancet Oncol 12(6): 575-582.

Wong J, Szumacher E (2012) Patients' decision-making in radiation oncology. Expert Rev Pharmacoecon Outcomes Res 12(1): 95-104.

cc)(i)(2) This work is licensed under the Creative Commons cy NG SA Attribution-NonCommercial-Share Alike 3.0 Unported License. To view a copy of this license, visit http://creativecommons. org/licenses/by-nc-sa/3.0/ 